\title{
Proyectos globalizados. Cómo articular la innovación en Educación Secundaria
}

\author{
Global projects. How to set up innovation processes in Secondary Education
}

\author{
$\mathbf{M}^{\mathrm{a}}$ José Pérez Albo \\ e-mail: mariajose.pereza@uam.es \\ Universidad Autónoma de Madrid \& CEIPS Santo Domingo. España
}

\author{
Alicia Gutiérrez Lacalle \\ e-mail: secundariasantodomingo50@gmail.com \\ CEIPS Santo Domingo. España
}

\section{Resumen}

El siguiente artículo pretende mostrar los aspectos básicos sobre los que se asienta la innovación en la Educación Secundaria en un centro público de la Comunidad de Madrid. Para ello, tomamos marco los elementos clave de mejora señalados en la investigación. A lo largo del artículo destacaremos los proyectos globalizados, las estrategias de formación del profesorado, las vías para promover la colaboración entre docentes y otros factores que contribuyen a la innovación; una innovación que tiene como meta proporcionar al alumnado una educación de calidad y otorgarle un papel activo en su proceso de aprendizaje. Asimismo, se detallarán diferentes estrategias que se están llevando a cabo en nuestro centro para ofrecer una educación personalizada, inclusiva y de calidad. En este sentido, el desarrollo de proyectos globalizados constituye el principal motor de actuación, ya que permite integrar todas las piezas del engranaje educativo y relacionar los contenidos de las diferentes disciplinas. Además, da la posibilidad de reorganizar el uso de los espacios y tiempos de aprendizaje. Estas estrategias fomentan la consolidación de las competencias indispensables para el crecimiento integral de los estudiantes, lo que favorece, por un lado, la adquisición de conocimientos y, por otro, el desarrollo de la inteligencia emocional, las habilidades sociales, el trabajo en equipo, la actitud investigadora y crítica; en definitiva, soft skills que serán determinantes para el crecimiento académico y profesional del alumnado.

Palabras clave: innovación educativa; mejora de la escuela; enseñanza secundaria; aprendizaje basado en proyectos.

\section{Abstract}

This paper aims to show the main elements on which innovation processes are based at a public Secondary school in Madrid, built up from the key elements found by the educational research. Along the description, we will point out some common issues such as global projects, several ways for teacher's training, vias to promote collaboration between teachers of different areas and many other items which contribute to carry on innovation processes in order to give students a high quality education and an active role in their learning process. We will also explain different strategic approaches that are currently being carried on to set up a personalized, inclusive and quality education process. Regarding that, globalized projects become a driving force that facilitates us to join together all the elements of the educational structure and to connect the various contents of different subjects, as well as enable the reorganization of learning spaces and times. Those strategies promote the consolidation of the main competences needed to achieve a student's global growth, leading not only to the acquisition of knowledge but also to the development of emotional intelligence, social abilities, team working, critical and investigating attitude, in the end, soft skills that will be decisive for the academic and professional development of the students.

Keywords: educational innovation; school improvement; secondary education; project based learning.

Recibido / Received: 16-01-2020

Aceptado / Accepted: 07-05-2020

Aceptado / Accepted: 01-07-2020

Cómo referenciar este artículo / How to reference this article:

Pérez Albo, M. J., \& Gutiérrez Lacalle, A. (2020). Proyectos globalizados. Cómo articular la innovación en Educación Secundaria. Tendencias Pedagógicas, 36, pp. 187-199. doi: 10.15366/tp2020.36.15 


\section{Introducción}

La pregunta acerca de qué elementos constituyen la clave para mejorar la calidad de un centro educativo y hacerlo más eficaz no es nueva. Desde los años 70 del siglo pasado, numerosas líneas de investigación han venido a señalar algunos aspectos que facilitan el cambio y la mejora en la escuela y han dado pie al desarrollo de estudios cada vez más complejos acerca de las piedras angulares de la transformación educativa (Stoll \& Fink, 1999; Murillo \& Krichesky, 2015). Paralelamente, hemos obtenido evidencias de que el aprendizaje es más profundo y efectivo cuando se realiza en red, protagonizando los propios procesos de indagación, contextualizándolo y aplicándolo (Hernández, 2017; Sancho, 2018).

Así las cosas, y en este camino de búsqueda constante de la mejora de la acción educativa, las últimas décadas han visto aflorar un buen número de cambios en los aspectos metodológicos, tratando de modificar la dinámica del aula y la interacción entre docentes y alumnado para conseguir un aprendizaje mayor y de más profundidad, modificando el rol de ambos para favorecer un papel más activo y generando modelos diferentes de acercamiento al conocimiento que buscan un aprendizaje más eficaz, de mayor impacto y con más solidez. Así han visto la luz y se han popularizado el aprendizaje basado en proyectos, el trabajo cooperativo, la clase al revés, la gamificación, los paisajes de aprendizaje y otras opciones de lo más variado y, desde luego, de diferente calado. Estas alternativas jalonan en estos momentos la actividad educativa en muchos contextos, pero no gozan del apoyo de todos los docentes y sectores: en algunos casos, porque no se ha probado suficientemente su eficacia; en otros, porque se aplican acríticamente sin generar cambios de profundidad y sin cuestionarse sus beneficios, su impacto y, sobre todo, el objetivo al que responden; y, además, porque a menudo se emplean simplemente como sustituto de metodologías más tradicionales y no se pone el foco en mejorarlas o complementarlas, situaciones que les hacen perder «adeptos»y, desde luego, oportunidades de mejora. Sin embargo, han tenido la virtualidad de estimular la reflexión en la práctica sobre el tipo de aprendizaje, de enseñanza y de alumnado que queremos y algunas se han instalado con solidez en el quehacer educativo.

La experimentación sobre estas metodologías activas ha tenido mucho eco en Educación Infantil y Primaria, favorecida en parte por las características de estas etapas, la preparación inicial de sus docentes, etc. En Educación Secundaria, aunque en los últimos años se ha hecho más frecuente hablar de diferentes metodologías, articular la respuesta se convierte en ocasiones, por su propia idiosincrasia, en una labor que abarca mucho más que al profesor en su aula y que requiere intervenir sobre aspectos y estructuras tan numerosas como diferentes para lograr que se convierta en una propuesta global que impregne realmente la cultura y la dinámica de un centro educativo.

En este artículo se desgranan algunos de los puntos de partida sobre los que se asienta la innovación educativa que se lleva a cabo en la Educación Secundaria en un centro público de la Comunidad de Madrid y se muestran proyectos recientemente desarrollados o en marcha en este momento, con el fin de dibujar un paisaje en el que se reflejen las piedras de toque que constituyen la fortaleza de la propuesta. De este modo, los principios e ingredientes básicos de los procesos de innovación se materializan en una respuesta concreta adaptada a la singularidad de nuestro centro educativo.

En primer lugar, se realizan algunas consideraciones previas para contextualizar la innovación y reflexionar acerca del papel del equipo docente y del uso de las TIC y los espacios en ese proceso, para dar paso, a continuación, a enmarcar todo ello en el proyecto de centro. Desde ahí se abre la descripción de cuatro propuestas concretas que se han puesto en marcha, el análisis de algunos elementos que favorecen o que dificultan el desarrollo de este tipo de proyectos y el impacto que están teniendo en alumnos, docentes y en el conjunto del centro, antes de realizar algunas consideraciones finales que puedan orientar su organización en otros espacios y tiempos.

\section{Algunas reflexiones de partida}

\subsection{Primera idea. Es imprescindible apoyar la innovación en evidencias cientificas y experiencias contrastadas}

Tanto en la literatura científica como en el relato de las experiencias prácticas existen algunos ingredientes que habitualmente están presentes en los procesos innovadores y que se sitúan en el punto de mira del cambio y la mejora. 
La investigación realizada por Murillo y Martínez Garrido (2018) recoge los factores de aula encontrados de manera consistente en la investigación internacional a lo largo de los años: el clima de aula, la preparación y estructuración de las clases, las actividades didácticas, la gestión del tiempo, un feedback frecuente y, por supuesto, la evaluación. Todos ellos se han mostrado reiteradamente influyentes tanto en el rendimiento académico como en el desarrollo socioafectivo de los estudiantes.

Por otra parte, en lo que respecta al profesorado, también parece clara la presencia de algunos elementos de eficacia docente, entre los que se repiten el ambiente de aprendizaje, la preparación de las clases, la gestión del tiempo, la retroalimentación que se ofrece al alumnado y la evaluación; y, junto a los que destacamos, el ofrecimiento de un objetivo y sentido del aprendizaje, el modelado, la facilitación de que sean los propios alumnos quienes desarrollen estrategias para resolver los problemas que se plantean, la realización de preguntas ajustadas al nivel de los estudiantes y la creación de oportunidades para aplicar el aprendizaje (Kyriakides, Christoforou, \& Charalambous, 2013).

Sin embargo, es necesario tener presente que ni uno ni otro son barcos aislados navegando en solitario, sino que ambos se encuentran integrados en un sistema organizativo que los nutre y da cobijo. Es por ello por lo que, paulatinamente, la investigación ha ido haciendo más hincapié en las aportaciones de las instituciones educativas (y del entorno de cada una de ellas) a la mejora y a la puesta en marcha de procesos de cambio e innovación. Entre los factores de centro más relevantes aparecen desde hace tiempo el liderazgo, la existencia de una visión y unos objetivos compartidos, un clima que favorezca el aprendizaje, las altas expectativas sobre el alumnado, la supervisión frecuente y retroalimentación de su trabajo, la relación con el entorno y la perspectiva de los centros educativos como organizaciones que aprenden (Murillo \& Muñoz-Repiso, 2002). Y, desde hace unos años, se están realizando investigaciones con modelos multinivel que tienen en cuenta los efectos de los diferentes niveles (contexto, centro, aula, alumnado) sobre el aprendizaje, ofreciendo una visión sistémica que permite trabajar teniendo en cuenta no solo las aportaciones de cada nivel sino las potencialidades de la interacción entre ellos.

Tenemos sólida evidencia, por tanto, de los puntos clave que nos permiten implementar cambios que den lugar a mejores propuestas educativas.

\subsection{Segunda idea. Cada centro imprime su propio sello innovador, cada uno debe encontrar su propia manera de articular la innovación}

A partir de la evidencia anterior surge también la necesidad de personalizar la innovación y ajustar las propuestas en función del contexto en el que se llevan a cabo. Son numerosos los trabajos que reflejan la importancia de realizar un análisis de la singularidad de cada contexto y partir de la presión interna para poder mejorar e introducir cambios de cierta relevancia (Bolívar, 2005). No existen fórmulas unívocas ni universales, sino que cada escuela debe organizar su propia solución de acuerdo con sus necesidades, su situación particular, su visión del centro en que quieren convertirse... De tal forma, el cambio y la mejora en un centro educativo deben ser un proceso planificado que responda a una manera concreta de entender la enseñanza y el aprendizaje y que tenga claras sus metas, más allá de la simple intención de implementar estrategias que generen nuevas dinámicas o de aplicar de forma acrítica unas u otras metodologías. En palabras de Gros y Lara (2009, p. 227):

«La innovación no puede ser una práctica aislada. Un proyecto de innovación responde a una necesidad de diferenciación estratégica. La diferenciación es la meta y la innovación es el proceso para conseguirla. Por consiguiente, para que una organización sea innovadora ha de sistematizar y ejecutar de forma consciente y controlada su desarrollo estratégico.»

Por eso, durante toda la exposición nos vamos a referir en paralelo a las evidencias que ofrece la literatura científica y a la manera concreta en que articulamos la innovación en nuestro centro, señalando los ingredientes que consideramos esenciales para que funcione en nuestro caso (que podría no ser el de otros, o sí...). Quizá no están todos los que son, pero todos los que vamos a señalar son esenciales para que llegue a buen puerto. 


\subsection{Tercera idea. Los docentes son el motor principal del cambio. Pero el cambio lo cambia todo}

A menudo nos encontramos con la creencia asentada de que el profesorado y el aula son los elementos claves para el cambio y la innovación, y resulta innegable que los docentes constituyen el eje sobre el que pivotan las iniciativas que se ponen en marcha en cualquier centro. Por tanto, resulta fundamental cuidar el compromiso, la colaboración y la capacitación del profesorado para llevarlas a cabo. Pero, además, tenemos la certeza de que no es posible la innovación y la mejora sin el trabajo en red, que se ha demostrado esencial no solo en la implementación de iniciativas sino en su sostenibilidad y su consolidación en el tiempo (Sloep \& Berlanga, 2011). En este sentido, Hargreaves y Fullan (2014) destacan la complementariedad de la mejora individual con el progreso colectivo y señalan la necesidad de crear una cultura colaborativa como base para un efectivo desempeño profesional; a través de ella, docentes comprometidos y en formación continua planifican y trabajan en equipo y ponen todo su potencial, recursos y experiencia al servicio del desarrollo organizacional, haciéndolo más efectivo y transformador y facilitando una mejora más sostenible, ya que su mejor desempeño permite «mejorar como individuo, aumentar el rendimiento del equipo e incrementar la calidad en toda la profesión» (2014, p. 47).

La primera actuación que se realiza en este sentido en nuestro centro es una formación inicial intensiva en los primeros días del curso, antes de que empiecen las clases, tanto para mostrar el planteamiento pedagógico y las bases metodológicas del proyecto como para iniciarse en las herramientas tecnológicas que se utilizan habitualmente en el aula y en el centro (cada profesor dispone de un iPad que contiene todas las aplicaciones y recursos necesarios para el trabajo, ya que no hay libros de texto). Aquí nos encontramos con una característica del centro que supone a la vez ventaja e inconveniente: somos un centro pequeño y estamos lejos de casi todo, y eso provoca que tengamos muchos profesores compartidos y demasiada inestabilidad del profesorado, aunque también hace más fácil la coordinación y el acompañamiento de profesorado veterano a quienes se incorporan al centro por primera vez.

Otra de las necesidades que queremos cubrir es la habitual falta de espacios y tiempos de coordinación en la Educación Secundaria. Nosotros buscamos esos tiempos y espacios mezclando grupos, organizando zonas de trabajo de docentes en pasillos, entradas... para trabajar juntos o simplemente para estar juntos. También se delegan responsabilidades, de manera que no es solo el equipo directivo quien inicia los proyectos y los difunde, sino que se responsabiliza a algunos docentes de determinadas tareas (p.e. la creación de vídeos o el programa de radio) y son ellos quienes se encargan de transmitir las necesidades y oportunidades al resto de los compañeros. Además, empleamos varias herramientas tecnológicas para organizar el trabajo, coordinarnos y saber quién está en cada proyecto en cada momento (el planificador de Trello, tableros de padlet, calendarios compartidos, la web...).

Todas estas pequeñas acciones afectan a cada docente individualmente, pero, sobre todo, van configurando un clima de aprendizaje concreto, una cultura de centro, una estructura organizativa que se asume como una forma específica y singular de trabajar, de enseñar, de aprender. Así, la innovación provoca cambios en las estructuras, en la manera de relacionarse y colaborar, en los tiempos, en la formación y capacitación del profesorado, etc. y el centro adquiere poco a poco una tendencia natural a la innovación, un ambiente de cambio que se cuela en la cultura y el clima de la escuela y genera a su vez mayor capacidad de innovación no solo en personas concretas sino en la organización en su conjunto, que aprende y se desarrolla (Gil, Antelm, \& Cacheiro, 2018).

Queda patente, pues, que un elemento intrínseco a nuestra manera de entender la innovación y la acción educativa es el trabajo en equipo. Sea cual sea el proyecto, el éxito y el trabajo son de todos, independientemente del origen de las ideas. Hacemos todo lo posible por crear un clima de trabajo en común en el que se comparta el conocimiento y en el que sea posible el apoyo y el asesoramiento del equipo directivo, del departamento de orientación y del resto del profesorado de cualquier área y nivel educativo, para que cada docente encuentre la forma con la que integrarse en los proyectos que se inician y la manera de aplicarlo en su área de conocimiento. Por eso, también se da cabida a las propuestas del profesorado: a veces se reorienta dentro del planteamiento general del centro y en otras ocasiones simplemente se intenta apoyar al docente para que se pueda llevar a cabo su iniciativa, favoreciendo con ello su implicación en el centro y su compromiso con el proyecto.

En este aspecto, el trabajo conjunto de las estructuras de coordinación existentes en el centro es fundamental. Los departamentos (en muchos casos, «mini-departamentos» o departamentos unipersonales) se coordinan para ir dando forma a las propuestas iniciales, y también la estrecha colaboración entre 
el Departamento de Orientación y el Equipo Directivo constituye un excelente cauce para difundir y dar cabida a las propuestas y hacer de enlace de los agentes internos entre sí y con otros recursos externos. No se olvida tampoco a las familias, a las que se informa e involucra frecuentemente en los proyectos que se desarrollan.

No podemos cerrar este aspecto sin hacer referencia al liderazgo, que la investigación lleva señalando desde hace décadas como un elemento indispensable en la mejora de la escuela y en el mayor rendimiento del alumnado (véanse, por ejemplo, la síntesis clásica de Stoll \& Fink, 1999; la revisión teórica y práctica coordinada por Murillo \& Muñoz-Repiso, 2002, o las aportaciones más recientes de Sun \& Leithwood, 2014; Harris, 2013; y Hargreaves \& Fullan, 2014). Esta tarea se ejerce con mayor satisfacción cuando puede llevarse a cabo una labor de liderazgo pedagógico y fomentar una cultura de colaboración (tal como señala el reciente trabajo de Martínez \& Gil, 2018), favoreciendo espacios de trabajo en común y potenciando la actuación de los centros educativos como verdaderas comunidades profesionales de aprendizaje. En nuestro caso, desde el equipo directivo se ejerce el liderazgo de manera firme, colaborativa y participativa, orientando las iniciativas en la línea del proyecto del centro, ofreciendo cauces para desarrollar las ideas del profesorado y estableciendo mecanismos para crear un ambiente de colaboración y satisfacción profesional en el centro que sin duda contribuye, como si fuera una dinamo, a mantener en movimiento a la comunidad educativa en la dirección de la mejora.

\subsection{Cuarta idea. El cambio debe afectar a todos los alumnos y modifica el uso de los espacios y tiempos}

Hasta el momento tenemos bases sólidas, ideas adaptadas a un contexto singular y vías de impulso y colaboración. Pero todo esto se desarrolla en un lugar y tiempo concretos y con unos alumnos determinados, por lo que la siguiente reflexión es ¿cómo hacemos para que todos los estudiantes se vean incluidos y beneficiados por el cambio que proponemos? ¿qué tiene esta nueva estructura que ofrecerles a todos? ¿dónde y cuándo la vamos a llevar a cabo?

Podría parecer una perogrullada afirmar que el cambio debe ser para todo el alumnado o no será y que ninguna propuesta metodológica puede realizarse sin estar orientada a la inclusión, pero cuantos nos dedicamos a la educación desde cualquiera de sus lugares sabemos que muchas veces no es así. Las tendencias de los últimos años están poniendo de relieve, tanto desde la investigación como desde el quehacer práctico, la importancia de ofrecer un currículo diseñado desde el principio para todos los alumnos y alumnas y de que los responsables y el profesorado se comprometan con la inclusión, evitando las políticas y las prácticas segregadoras (Ainscow, Dyson, \& Weiner, 2013; Escudero \& Martínez, 2011). Es necesario realizar un planteamiento flexible, funcional e integrador, crear un entorno inclusivo y plantear un Diseño Universal del Aprendizaje que proporcione diferentes medios de representación y comprensión de los contenidos, combine distintas habilidades, ofrezca opciones para desarrollar las funciones ejecutivas y de planificación y, por último, que muestre variados medios para favorecer la motivación, el esfuerzo y la autorregulación del alumnado (Roselló, 2010; CAST, 2011). Por eso, la fórmula de proyectos globalizados ofrece la posibilidad de realizar actividades en diferentes niveles (reproducir, clasificar, analizar, evaluar, crear...) y con distintos tipos de habilidades y competencias, dando cabida a cada uno de nuestros estudiantes dentro de un currículo común y del aula ordinaria.

En esta perspectiva inclusiva no podemos desdeñar la importancia que ocupan el espacio en el que se desarrolla el aprendizaje y la distribución del tiempo en el aula, y ambas variables están asociadas a la organización de la tarea y también a las relaciones entre los diferentes actores educativos.

La realización de proyectos globalizados, en el marco de un proyecto de centro ensimismado en el proceso activo y creador del alumnado, hace necesario modificar tanto la estructura académica como la estructura social del aula, generando un nuevo sistema de interacciones centrado en el trabajo que disminuye las disrupciones y crea un clima de aprendizaje favorable. Es decir, no se trata solo de cambiar la forma de acercarse al conocimiento y gestionarlo, sino que esto lleva indefectiblemente a producir un sistema de relaciones en el aula de marcado carácter cooperativo, más organizadas (aunque, al tiempo, más autónomas) y más colaborativas. Así, trabajar en equipo conlleva per se una organización distinta de los tiempos y un uso concreto del espacio, ya que requiere una disposición del aula, de los momentos y de los materiales que no está centrada en el docente y su explicación sino en el grupo y su tarea. 
Por un lado, la gestión del tiempo, que se ha revelado en numerosos estudios como factor de eficacia docente que ejerce una indudable influencia en el rendimiento (Murillo \& Martínez-Garrido, 2018), se optimiza al utilizar los proyectos globalizados, al menos así ha resultado en nuestro caso. En este tipo de trabajos, los estudiantes están más motivados, se reduce el tiempo que se tarda en empezar la clase (a veces incluso se suceden dos clases sin transición), se realiza una organización de la tarea al inicio de cada sesión que distribuye más eficazmente el tiempo de la misma y se da continuidad al trabajo que se ha hecho en otras sesiones y asignaturas, aumentando el tiempo disponible para que el alumnado investigue y aprenda y respetando en mayor medida su ritmo de aprendizaje.

Por otro lado, el uso de espacios cambia y, a su vez, hace cambiar las relaciones e interacciones que se establecen. Autores como Prakash Nair (2013) destacan cómo el diseño de espacios más centrados en el estudiante permite ajustarse mejor a las necesidades de éste y aumenta las posibilidades educativas del espacio, de forma que se favorece un aprendizaje mucho más competencial, al tiempo que se maximiza la versatilidad del espacio educativo disponible.

En esta línea, debemos recordar en primer lugar que lo que debe condicionar el uso del espacio no es su organización previa, sino las necesidades que tenemos: es el espacio el que debe adaptarse a los usos que nosotros necesitamos, y no al revés. Por otra parte, como señala Nair, el espacio debe ser acogedor, versátil, crear un clima positivo y permitir múltiples actividades; y, en línea con las directrices del Diseño Universal del Aprendizaje, debe ser además inclusivo. Es fundamental, asimismo, que el espacio favorezca el aprendizaje en red, para dar lugar al aprendizaje cooperativo del alumnado y también para que la institución educativa pueda convertirse en una comunidad profesional de aprendizaje y dé cabida además a la presencia de otros agentes más lejanos.

Finalmente, ningún cambio en este momento puede quedar al margen las tecnologías, pero su presencia y posibilidades no deben ser sobredimensionadas y acapararlo todo. De esta forma, aunque algunas propuestas pasan por equipar los espacios con recursos, materiales y puntos de acceso para plataformas digitales y dispositivos móviles, es imprescindible contemplar la necesidad de adaptar y utilizar unos recursos que propicien la interacción personal, que los conviertan en espacios sociales de aprendizaje.

En definitiva, la reflexión sobre los espacios debe estar siempre mirada desde la perspectiva de la flexibilidad y la innovación, como señala Siro López (2018), al servicio de los objetivos de aprendizaje y teniendo en cuenta cómo concebimos el aprendizaje, y no pueden pensarse al margen de estas dos consideraciones. Por eso, en el centro hemos optado por abrir las puertas del aula y favorecer la posibilidad de que cualquier espacio pueda convertirse en un lugar de aprendizaje, de manera que es frecuente ver grupos de alumnos trabajando en espacios habilitados en los pasillos o en despachos, creando sus propios espacios de trabajo en diferentes lugares y organizando el mobiliario y los materiales del aula en función de las necesidades que se presentan en cada momento. La colaboración entre docentes, en este contexto, se maximiza, permitiendo incluso algunos espacios de docencia compartida que multiplican las oportunidades de aprendizaje del alumnado, favorecen la personalización y contribuyen a la mejora profesional docente, así como a la innovación y la inclusión (Ainscow, 2016; Durán, 2019).

\section{Cómo se integra la propuesta en el proyecto global de centro}

Como ya hemos señalado, la actuación del profesorado no puede permanecer aislada, sino que se encuentra inmersa en la organización educativa y, para ser eficaz y sostenible en el tiempo, necesita articularse en torno a una visión clara y unos objetivos compartidos por toda la comunidad. Y nuestro caso no es una excepción.

El nuestro es un centro innovador desde el punto de vista tecnológico, pero sobre todo orienta la innovación a conseguir dar a cada uno el lugar que necesita, desarrollar al máximo sus posibilidades y aprovechar sus talentos y potencialidades; esto es lo que constituye la seña de identidad del proyecto de Secundaria en el centro. ¿Qué herramientas usamos en este proceso?: la creatividad, el trabajo cooperativo, la comunicación, la incentivación y el desarrollo de las diferentes capacidades, todo ello englobado dentro del uso de metodologías activas que empoderen al alumnado para que sea el actor principal de su aprendizaje.

En nuestro caso se trata de una comunidad educativa cohesionada que se esfuerza por desarrollar la creatividad y las competencias comunicativas de nuestros alumnos. Los docentes pasan a ser guías en el proceso de enseñanza aprendizaje, acompañando de cerca a los estudiantes que utilizan las TIC 
para buscar, investigar, transformar, comunicar y compartir la información, de manera que consiguen un aprendizaje global. Nuestro proyecto impulsa el pensamiento crítico, la oratoria, la escritura creativa y la creación audiovisual como herramientas básicas. Impulsamos la investigación, la curiosidad y la generación de ideas que se plasman, posteriormente, en la creación de sus propios contenidos. Todo ello permite crear alumnos autónomos, responsables y críticos que se convertirán en ciudadanos activos en el futuro. Basamos nuestra propuesta en la comunicación porque creemos firmemente que los estudiantes son capaces de interiorizar y dominar los aprendizajes si son capaces de transmitirlos y comunicarlos con rigor. Los alumnos investigan los contenidos acompañados por sus profesores y, posteriormente, comunican lo aprendido mediante la creación multimedia. Para desarrollar la curiosidad y la motivación parten siempre de las ideas planteadas en un mapa mental inicial que se amplía después con la investigación del alumnado. Esta herramienta nos permite jerarquizar las ideas, facilitar la memorización y la relación de conceptos y fomenta la reflexión. Posteriormente, desarrollan los contenidos a través de artículos para el periódico, programas de radio y televisión y diversos proyectos globalizados que integran las TIC.

\title{
4. Por qué los proyectos globalizados
}

Hemos titulado este artículo bajo el paraguas de los proyectos globalizados porque esta manera de trabajar es respetuosa con los factores de relevancia para la mejora que hemos destacado y aúna muchos de los elementos que conforman nuestro sello particular y que garantizan el trabajo conjunto de toda la comunidad educativa dirigida hacia un fin común: la educación rigurosa y efectiva de nuestros estudiantes, a partir de enfocar la mejora en algunos elementos concretos del centro y del aula que pueden provocar el cambio (Bolívar, 2005).

Las ventajas del trabajo por proyectos han sido destacadas ya en numerosas ocasiones, ya que permite conectar con la realidad, crear experiencias educativas en las que el alumno sea co-artífice del aprendizaje y favorecer un aprendizaje experiencial, interdisciplinar e integrado, tal como ilustran autores como Juanjo Vergara (2015).

Pero, además, ayudan a no tropezar con una de las piedras con las que recurrentemente nos topamos al diseñar procesos de aprendizaje e innovación: la descontextualización del aprendizaje. Tal como ilustra Fernando Trujillo (2018, p. 81):

\begin{abstract}
«En la escuela con frecuencia nunca llegamos a jugar el partido. No es extraño aun ver que somos capaces de pedir a nuestros estudiantes que memoricen la definición del cuento sin atrevernos a crear o a representar uno en clase; o podemos encargarles que hagan decenas de ejercicios sobre estadísticas o probabilidad sin llegar nunca a realizar una investigación donde unas u otras sean necesarias; o podemos recorrer todo el espectro de las Ciencias de la Naturaleza sin pasar nunca por el laboratorio, realizar una observación al aire libre o desarrollar un sencillo experimento.»
\end{abstract}

Los proyectos globalizados, sin embargo, evitan esta tentación y hacen posible el aprendizaje contextualizado, interrelacionado, significativo y activo. Normalmente surgen de la necesidad de dar res-puesta a un problema o a una pregunta que se ha planteado en el aula o en el equipo docente, de forma que nacen en un escenario que tiene sentido y aplicación real y se sitúan en un contexto concreto.

También permiten realizar una evaluación más ajustada y completa de los aprendizajes adquiridos y las competencias desarrolladas. La mayor parte de los proyectos se integran dentro del currículo ordinario y se evalúan a través de rúbricas específicas diseñadas por el equipo docente. En muchos casos forman parte del procedimiento de evaluación ordinario de cada asignatura y, en otras ocasio-nes, se plantean como propuestas voluntarias de enriquecimiento personal.

Pero ¿cómo se organizan? A continuación, presentamos cuatro propuestas concretas desarrolladas estos últimos años en Educación Secundaria para mostrar la estructura que constituye su armazón y la fórmula organizativa con la que se articulan en la práctica. 


\subsection{Mujeres para la Historia}

Se trata de un proyecto globalizado que iniciamos hace cuatro cursos y tiene continuidad actualmente. Con él pretendemos que los alumnos de cualquier curso de la ESO tomen sus propias decisiones, investiguen y trabajen la información biográfica de cada mujer elegida para elaborar una estatuilla que recoja la muñeca, el vídeo y el dibujo homenaje a cada una de ellas. Se aborda, por tanto, desde diferentes asignaturas (Lengua y Literatura, Educación Física, Matemáticas, Biología, Tecnología, Plástica, Valores éticos...), orientando el trabajo de nuestros estudiantes hacia la realización de material didáctico propio y a que ellos mismos generen su propio contenido. Así, las metas de este proyecto se dirigen a desarrollar la capacidad de comprender las características de diferentes contextos históricos y cómo afectan a la vida cotidiana de sus habitantes, analizar el papel y las circunstancias de la mujer a lo largo de la Historia y desarrollar valores éticos expresados en los Derechos Humanos. Junto a estos objetivos se encuentra el fin siempre presente en nuestros proyectos de desarrollar la capacidad de creación, trabajando sobre procesos de elevada complejidad cognitiva siempre que es posible y de acuerdo con las potencialidades de cada alumno/a.

Los alumnos han diseñado con la impresora 3D un soporte que recoge la muñeca, donde se coloca una postal con el dibujo que cada una y un código QR que mostrará un vídeo elaborado por los alumnos y alumnas con la biografía de cada mujer. La comunicación de la información se transmite, por tanto, a través de distintos códigos y soportes.

Al margen de lo estrictamente curricular, Mujeres para la Historia tiene una clara proyección social. A través de la exposición y el trabajo previo, nuestros alumnos valoran y reflexionan sobre el papel de la mujer en distintos ámbitos de conocimiento a lo largo de la historia y acerca de su situación actual y futura en la sociedad.

Nuestro interés por que el proyecto traspase los muros físicos del centro nos ha llevado a realizar una exposición el 8 de marzo (con motivo del día de la mujer trabajadora) e invitar a las familias de los estudiantes, haciéndoles partícipes del proceso de enseñanza-aprendizaje de sus hijos, y a los ancianos que viven en una residencia cercana. Los alumnos han compartido con ellos su experiencia creadora y les han contado las historias de las diferentes mujeres protagonistas de la exposición. Esto nos ha llevado a desarrollar una interesantísima vinculación intergeneracional que les ha permitido compartir experiencias, intereses y vivencias.

Otra aportación clave del proyecto es la consecución de estrategias comunicativas para la vida real. Los estudiantes se han encargado de la difusión del evento, de diseñar las invitaciones, idear las diferentes maneras de hacérselas llegar a los invitados, organizar el evento, diseñar el cartel anunciador, actuar de anfitriones y recibir a compañeros, profesores, padres, ancianos, vecinos, etc.

Se trata, además, de una experiencia sostenible en el tiempo, ya que la exposición queda para los siguientes cursos e incluso la vamos ampliando año tras año con la incorporación de nuevos personajes.

\subsection{Inteligencia emocional}

En este proyecto hemos aunado aspectos académicos como la descripción, el dibujo y la creación audiovisual y tecnológica con cuestiones emocionales que nos ayudan a generar un mejor clima de convivencia, respeto, empatía y compañerismo. De este modo, a través del proyecto los estudiantes serán capaces de demostrar su comprensión de las características de los textos descriptivos (así como de elaborarlos adecuadamente), realizar composición de imágenes y diseñarlas en formato dibujo para después transformarlas y expresarlas utilizando diferentes soportes tecnológicos, reconocer las distintas emociones y su expresión en la propia persona y en los demás, así como comprender cómo se producen y expresan las emociones en distintos niveles de desarrollo.

Para ello facilitamos a los alumnos de $4^{\circ} \mathrm{ESO}$ un listado de emociones, algunas de ellas antitéticas, para que las investiguen y describan. Esto conlleva una reflexión profunda sobre las emociones propias y ajenas que son, sin duda, un reflejo claro de nuestro cuerpo y mente.

Una vez que hemos trabajado la importancia y características de las distintas emociones, los estudiantes han diseñado unas cartas que contienen la definición propuesta por ellos mismos y la imagen que han dibujado. En la parte trasera de cada carta aparece un código QR que deriva al vídeo explicativo de cada emoción. De este modo hemos creado, entre todos, una baraja de emociones. 
Posteriormente, los estudiantes han propuesto juegos de mímica, cuentos, juegos tipo trivial, etc. para poder utilizar la baraja en las diferentes etapas educativas, desde Infantil hasta Secundaria. Para adecuar los juegos al momento evolutivo de cada nivel, los estudiantes de $4^{\circ} \mathrm{ESO}$ han tenido que investigar cómo son los niños en cada etapa, qué les gusta, cómo se sienten...

Como no queríamos que el trabajo se quedase reducido a los juegos, editamos e imprimimos material con los dibujos y enlaces a los vídeos y los hemos puesto por las paredes y escaleras del centro, de modo que cualquiera que quiera investigar sobre una emoción pueda hacerlo enfocando con su i-Pad el código QR correspondiente.

\subsection{Los cinco reinos}

En este caso, se trata de un proyecto internivelar e interdisciplinar sobre el período medieval. Consiste en la elaboración, por parte del alumnado de $2^{\circ}$ de la ESO, de cinco reinos imaginarios inspirados en la realidad social, cultural y política del medievo, etapa histórica que se trabaja tanto en Lengua como en Geografía e Historia en ese nivel. Los objetivos de aprendizaje fundamentales de este proyecto se centran en que los estudiantes sean capaces de analizar las características de la época medieval, y de recrear mundos personales partiendo de modelos constatados de realidades históricas, musicales, literarias y tecnológicas, así como fomentar la búsqueda de soluciones en equipo y desarrollar la creatividad a través de diferentes soportes.

Trabajan conjuntamente los departamentos de Geografía e Historia, Lengua Castellana y Literatura, Dibujo, Música e Inglés. El proyecto se realiza en tres fases. En la primera, correspondiente al primer trimestre, el alumnado se distribuye en cinco grupos de seis personas cada uno. Se les propone la creación de cinco reinos sobre plano-maqueta con diferentes características y materiales. Todos ellos tendrán una historia y una forma de gobierno propia que inventarán y desarrollarán por escrito a través de leyendas, poemas épicos y genealogías que se recogerán en un códice. Los estudiantes también diseñarán un escudo y una bandera para cada reino y se elaborará un juego a través del cual se descubre que el equilibrio está en la unión de los reinos: todos los escudos configuran el gran mosaico identitario de la Tierra de Los Cinco Reinos. Los profesores de los diferentes departamentos recrearán figuras históricas para que los alumnos se familiaricen con ellos y recojan sus historias en el códice. En el segundo trimestre desarrollamos la segunda fase, en la que los estudiantes recrean un scriptorium y transcriben textos para el códice de los cinco reinos. En la tercera fase, realizada en la tercera evaluación, los cinco reinos reciben la visita de los personajes trabajados a lo largo del curso, encarnados por profesores y alumnos voluntarios, y finalizamos el proyecto con la realización de una feria medieval.

\subsection{Análisis del termómetro digital}

En este caso se trata de un proyecto que permite a nuestros alumnos indagar y desarrollar el método científico desde diferentes asignaturas y cursos, con el fin de que sean capaces de reconocer sus pasos, trabajar empleando sus principios y aplicarlo a la resolución de problemas y situaciones de la vida cotidiana.

La ciencia avanza planteándose cuestiones que preocupan e inquietan al ser humano y buscando soluciones a éstas. En nuestro caso, los interrogantes que nos llevaron a iniciar el proyecto surgen de un momento cotidiano en el que detectamos que un termómetro digital no está cumpliendo de manera óptima los fines para los que ha sido creado. La duda surge cuando colocamos un termómetro digital a dos personas de 72 años con posible fiebre y observamos que, en el momento en el que se emite el pitido, no son capaces de oírlo. Planteamos a los alumnos la posibilidad de que se tratase de algo puntual o que, por el contrario, afecte a más personas. El objetivo del proyecto es responder a la siguiente pregunta: ¿Y si realmente a determinada edad, por el deterioro propio de la misma, igual que la presbicia en la vista, dejamos de poder percibir determinadas frecuencias? ¿Cómo podríamos solucionar este problema?

El primer paso fue medir la frecuencia de emisión de los termómetros, que se encuentran sobre los $9000 \mathrm{~Hz}$, e investigar sobre la pérdida de audición con la edad, comprobando que primero dejamos de percibir las frecuencias agudas y por último las graves. Comprobamos la incidencia de este fenómeno en una muestra de población entre los 65 y 100 años. Para ello entramos en contacto con una residencia de ancianos próxima al Instituto, que accedió a que realizáramos el muestreo entre los ancianos que viven allí. Procedimos a tener una entrevista personal para comunicarles el proyecto, 
realizar las audiometrías con una aplicación que los alumnos descargaron en sus dispositivos móviles y posteriormente se realizó el estudio por franjas de edades. Un grupo de estudiantes se encargó de recoger material audiovisual para realizar un documento sobre el estudio. Implicamos a todos los niveles educativos y a diversos departamentos, de manera que los alumnos de $1^{\circ} \mathrm{ESO}$ se encargan del muestreo, de buscar la aplicación que más se adapte a las necesidades de la audiometría, probando varias y analizando aquellas que deben descartarse y el motivo por el que no son válidas, favoreciendo, de este modo, el razonamiento crítico y el método científico. Los alumnos de $4^{\circ}$ de ESO reciben el muestreo y se ocupan de hacer el análisis estadístico, aportando datos, tablas, gráficas que puedan darnos respuesta a nuestras preguntas. Los alumnos de $3^{\circ}$ de ESO se dedican a estudiar el oído, aspecto que aparece dentro del temario de Biología, y aportan datos precisos sobre cómo se producen el mecanismo de audición y la pérdida de audición con la edad. Los alumnos de $2^{\circ}$, partiendo de las conclusiones, realizan prototipos alternativos a los modelos que hoy existen en el mercado, siguiendo estas líneas de investigación: el termómetro que vibra como los móviles a la vez que emite el sonido, una pulsera en la muñeca que emita luz una vez que suene el termómetro, una pulsera que vibre y otras sugerencias creativas de los alumnos.

Finalizada la investigación, es importante que los alumnos entiendan la importancia de la difusión de ideas y la divulgación científica. Para ello, los alumnos de $1^{\circ}$ de ESO buscan vías para poder dar a conocer su estudio y de esta forma fomentar que se produzca el cambio. Pueden divulgar su trabajo, analizar qué organizaciones pueden permitir realizarla, transmitir los resultados a empresas que se encargan de fabricar los termómetros para que modifiquen la frecuencia, etc. En el desarrollo del proyecto han intervenido los departamentos de Matemáticas, Lengua Castellana y Literatura, Tecnología y Biología.

\section{Dificultades y puntos de apoyo que encontramos en el camino}

A lo largo de la realización de los cuatro proyectos mencionados hemos podido constatar que reiteradamente nos cruzamos con algunas circunstancias que jalonan de OBSTACULOS nuestro camino.

$\mathrm{El}$ primero es, sin duda, la inestabilidad del profesorado. $\mathrm{Al}$ ser un centro pequeño y en crecimiento, la mayor parte de los docentes de secundaria son interinos y ninguno es definitivo, por lo que el cambio es constante y esta circunstancia hace muy complicado dar continuidad a los proyectos, dificulta la organización de los equipos docentes, que se renuevan en gran parte cada año, y tampoco permite rentabilizar la formación ofrecida al profesorado y la cultura de centro que nos esforzamos en crear cada curso.

Precisamente esta necesidad de formación continua acerca de los aspectos didácticos y metodológicos, que sigue siendo un problema sin resolver en el acceso a la docencia en estas etapas, es otro de los temas candentes cada año.

Ninguna de estas dos circunstancias nos ayuda a mantener la idea de cambio y la visión que tenemos del centro, ya que frecuentemente nos vemos impelidos a reforzar esa visión y a esforzarnos por transmitir la manera de entender la innovación, el aprendizaje, el papel del docente... Tampoco ayuda el individualismo tradicional de la función docente, acostumbrado a trabajar de manera aislada e independiente en su aula.

Junto con ello, nos encontramos con una clara falta de espacios, que cada curso es mayor porque el centro crece, pero los espacios de los que disponemos no lo hacen a la par que el alumnado. Esto nos ha obligado a reinventarnos, pero también nos encorseta, igual que lo hace la rigidez de los horarios de la Educación Secundaria, que nos gustaría poder modificar para ser más eficaces.

En el otro lado, también nos encontramos con ELEMENTOS FACILITADORES del cambio en nuestra escuela.

Un centro pequeño dificulta la estabilidad de las plantillas, pero hace más fácil la coordinación del profesorado, ya que el equipo docente es poco numeroso y se encuentra concentrado en un número reducido de aulas. Así, la mayoría conocemos lo que hacen los compañeros y es fácil coincidir con ellos para organizar y planificar las distintas actuaciones.

Por otro lado, el acceso cotidiano y el uso habitual de las Tecnologias de la Información y la Comunicación facilita la organización y la ejecución de los proyectos, ya que ofrece un espacio virtual en el que aglutinar los materiales y procesos que se van realizando a lo largo de los proyectos.

Al tratarse de un centro público que ofrece desde Educación Infantil hasta Educación Secundaria Obligatoria, parte de los estudiantes (aquellos que han cursado la etapa anterior en nuestro centro) 
vienen de la Educación Primaria acostumbrados a trabajar con estas herramientas y estrategias, de manera que al llegar a la ESO están familiarizados con esta forma de trabajo.

Igualmente, la predisposición natural de los alumnos a aceptar el trabajo a través de las TIC y el interés por trabajar de forma cooperativa hacen que sea más sencillo plantear a los alumnos proyectos globalizados y que los asuman con naturalidad e ilusión, incluso cuando se trata de retos voluntarios.

La facilidad de acceso a la información también hace que sea más fácil trabajar de esta manera, ya que nuestros estudiantes pueden acceder a diferentes fuentes con prontitud y la diversidad de estas nos sirve sobremanera para desarrollar su espíritu crítico y su razonamiento y creatividad.

Por último, la duplicidad y complementariedad de algunos contenidos de los temarios oficiales en diferentes áreas también contribuye a que trabajen juntos varios departamentos, como ocurre, por ejemplo, con Lengua Castellana y Literatura y Geografía e Historia o Tecnología y Matemáticas o Geografía y Biología.

\section{Una breve conclusión y algunas pinceladas para iluminar el paisaje}

A lo largo de este artículo hemos ido dando voz a los elementos que constituyen parte de nuestra manera de llevar a cabo la innovación en un centro educativo concreto, siempre con la mirada puesta en ofrecer mayores y mejores oportunidades de aprendizaje a todos nuestros alumnos y alumnas. Hemos partido de la propuesta de los proyectos globalizados como eje para describir experiencias concretas que pueden resultar valiosas en sí mismas, pero que en realidad son también la excusa tras la que se esconden aspectos vertebradores de una acción educativa dirigida a la mejora y enfocada a un aprendizaje verdadero y de calidad.

Las acciones puestas en marcha tienen un IMPACTO directo e indirecto tanto en el aula (en alumnos y docentes) como en la etapa en su conjunto. Así, utilizar los proyectos globalizados nos lleva a establecer nuevos vínculos y maneras de relación entre los alumnos y de estos con el profesorado, favoreciendo la colaboración entre grupos y niveles educativos y permitiendo que el alumnado sea un actor activo en el proceso de enseñanza-aprendizaje. Esto permite trabajar su autonomía, creatividad y habilidad comunicativa de una manera aplicada y funcional que da sentido a los aprendizajes e integra los contenidos de las diferentes áreas de conocimiento. Al mismo tiempo, en la línea de trabajo competencial de la que hablábamos al principio, y con las aportaciones de profesorado que viene de centros y entornos muy diferentes, venimos constatando que esta forma de trabajo ayuda al alumnado a desenvolverse de manera más autónoma y auto-regulada en el aula, a trabajar en equipo, respetando a todos los compañeros y aprendiendo a integrar las aportaciones de todos, a hacer una escucha activa en clase en vez de permanecer pasivamente en ella y, por último, contribuye a que aprendan a hacer un uso de las tecnologías para el estudio y el trabajo en el aula y no solo para el ocio y el entretenimiento, fomentando su capacidad crítica de selección de la información, la búsqueda de datos y fuentes solventes y el manejo diestro de los recursos tecnológicos. Pero, además, educa al alumnado en el valor de la diversidad, puesto que les enseña a vivirla como un aspecto natural, a valorar los talentos del otro y a integrar a todos los compañeros en el equipo, sacando a la luz las fortalezas de cada uno y ofreciendo a cada estudiante una posibilidad para desarrollarse y trabajar los conocimientos que se abordan dentro del currículo, convirtiéndose en un potente impulsor de la inclusión.

Sin embargo, no es beneficioso únicamente para los estudiantes, sino que tiene un impacto duradero y profundo sobre el profesorado, que ve potenciado su desarrollo profesional y adquiere nuevas herramientas con las que mejorar su competencia didáctica y pedagógica. Esta forma de trabajo aumenta su formación y su capacidad para el uso de elementos tecnológicos, proporciona más recursos para atender a la diversidad, mejora sus técnicas didácticas y permite conjugar diferentes estrategias metodológicas y acercarse a ellas de manera natural, comprobando en la propia acción educativa las ventajas y dificultades que conllevan. Por otro lado, gracias la realización de estos proyectos interdisciplinares, los docentes de Secundaria participan más y más activamente en procesos de reflexión colaborativa sobre la propia práctica y encuentran nuevas estrategias para comunicar los conocimientos de su disciplina. El empleo de proyectos globalizados y el esfuerzo por crear un clima de colaboración centrado en el aprendizaje logra que sean (seamos) más capaces de trabajar con los demás y de enriquecernos con las aportaciones de los compañeros, mejora la capacidad de trabajo en equipo, la confianza en el trabajo de los compañeros y la coordinación, así como las relaciones interpersonales. Y, finalmente, facilita un seguimiento frecuente del progreso del alumnado y lo hace sobre más 
competencias y capacidades, ya que las rúbricas de evaluación integran diversos tipos de aprendizajes y productos esperados e incorporan aspectos no solo del desarrollo cognitivo sino de las dimensiones social y personal.

Se va conformando así poco a poco una cultura de centro, junto con una estructura organizativa específica, que ponen en valor el trabajo del centro como comunidad de aprendizaje que se mueve orientada hacia el cambio.

En otro orden de cosas, esta estrategia que planteamos es sostenible en el tiempo, es fácilmente transferible a diferentes asignaturas y cursos y genera una forma de trabajar que se transmite e implica a los diferentes agentes educativos. Al integrar los diferentes contenidos y procesos académicos, resulta altamente replicable, contagiando e involucrando de manera natural a otros profesores y departamentos. Asimismo, permite realizar un uso más eficiente y productivo de los espacios, favoreciendo la sostenibilidad e integrando lugares comunes dentro y fuera del centro que a priori no están planteados como zonas docentes.

¿Cuáles son en este momento los aportes que necesitamos y los RETOS que nos planteamos para el futuro?

En primer lugar, consideramos que es imprescindible la formación del profesorado respecto a las cuestiones didácticas específicas de cada materia y acerca de las alternativas metodológicas más adecuadas para cada caso y, especialmente, para lograr una adecuada atención a la diversidad del alumnado. Esto, que sin duda debe ofrecerse en la capacitación inicial que da acceso a la función docente, también debería encontrar su momento y su lugar en la formación durante el desempeño de la profesión y en las actividades de la práctica diaria, por difícil que resulte encajarla en esta tesitura.

Sería más que deseable, a nuestro juicio, favorecer una cierta estabilidad del profesorado o, si no fuera posible, explorar vías para dar continuidad a los proyectos, aunque parte del profesorado no permanezca en el centro en años sucesivos. En relación con esto, sería más que interesante profundizar en la docencia compartida como estrategia de apoyo entre el profesorado y de mejora del proceso de enseñanza-aprendizaje.

Por otro lado, debemos seguir trabajando sobre los espacios y tiempos, insistiendo en el uso de nuevos espacios o en nuevas maneras de utilizar los ya existentes, así como necesariamente se nos impone explorar las posibilidades de flexibilizar los horarios y adaptarlos a las necesidades del alumnado y de los objetivos de aprendizaje.

Finalmente, tenemos por delante el reto de difundir los proyectos y colaborar con otros centros, creando redes de aprendizaje y conocimiento en las que aportar nuestra experiencia y con las que seguir creciendo.

La innovación no puede convertirse en una moda, en un requerimiento social a unas escuelas que a veces parecemos más obsesionadas por innovar a toda costa en una loca carrera por atraer alumnos, mantener una buena reputación y estar a la última que centradas en el aprendizaje y el crecimiento de nuestros estudiantes, que constituye el fin último de nuestro quehacer. Por el contrario, debe hacerse de forma planificada, sustentada en evidencias, coordinada y con una visión definida del lugar al que pretendemos llegar y para qué. Por tanto, sea cual sea la estrategia que se utilice, lo relevante es articular un proceso de mejora sistemático, sistémico, autocrítico y colaborativo que tenga claro hacia dónde se dirige.

El lugar natural de la innovación parece el aula, pero en realidad no puede producirse realmente si no permea al resto de estructuras y procesos del centro. El cambio metodológico modifica también necesariamente la evaluación y ofrece un enfoque mucho más competencial, que cambia la perspectiva sobre el currículo y sobre las interacciones que se producen en el proceso educativo y remueve la organización del centro, con la mirada puesta en la idea con la que abríamos este trabajo: ofrecer una educación de calidad en la que cada estudiante pueda encontrar el lugar que necesita.

\section{Referencias}

Ainscow, M. (2016). Diversity and Equity: A Global Education Challenge. New Zealand Journal of Educational Studies, 51(2), pp. 143-155. doi: 10.1007/s40841-016-0056-x

Ainscow, M., Dyson, A., \& Weiner, S. (2013). From exclusion to inclusion: a review international literature on ways of responding to students with special educational needs in schools. En-clave pedagógica: Revista Internacional de Investigación e Innovación Educativa, 13, pp. 13-30. Recuperado de 
https://rabida.uhu.es/dspace/bitstream/handle/10272/8169/De_la_exclusion_a_la_inclusion.pdf?sequence $=2$

Bolívar, A. (2005). Dónde situar los esfuerzos de mejora. Educaşão \& Sociedade, 26(92), pp. 859-888. doi: 10.1590/S0101-73302005000300008

CAST (2011). Universal Design for Learning Guidelines. Version 2.0. Wakefield, MA: Author.

Durán, D. (2019). Aprendizaje docente entre iguales: maestros y escuelas que aprenden unos de otros. Ámbitos de Psicopedagogía y Orientación, 50, pp. 50-62. doi: 10.32093/ambits.v0i50.1219

Escudero, J., \& Martínez, B. (2011). Educación inclusiva y cambio escolar. Revista Iberoamericana de Educación, 55, pp. 85-105. doi: 10.35362/rie550526

Gil, A. J., Antelm, A. M., \& Cacheiro, M. L. (2018). Análisis de la capacidad de innovación escolar desde la perspectiva del profesorado de educación secundaria. La escuela como organización que aprende. Revista Educar, 54(2), pp. 449-468. doi: 10.5565/rev/educar.864

Gros, B., \& Lara, P. (2009). Estrategias de innovación en la educación superior: el caso de la Universitat Oberta de Catalunya. Revista Iberoamericana de Educación, 49, pp. 223-245. doi: $10.35362 /$ rie490681

Hargreaves, A., \& Fullan, M. (2014). Capital profesional. Madrid: Morata.

Harris, A. (2013). Distributed leadership: Friend or foe? Educational Management Administration and Leadership, 41(5), pp. 545-554. doi: 10.1177/1741143213497635

Hernández, F. (Coord.). (2017). ¡Y luego dicen que la escuela pública no funciona! Investigar con los jóvenes sobre cómo transitan y aprenden dentro y fuera de los centros de secundaria. Barcelona: Octaedro.

Kyriakides, L., Christoforou, C., \& Charalambous, C.Y. (2013). What matters for student learning outcomes: A meta-analysis of studies exploring factors of effective teaching. Teaching and Teacher Education, 36, 143-152. doi: 10.1016/j.tate.2013.07.010

López, S. (2018). Diseño de espacios educativos. Aprendizaje y Creatividad. Madrid: Ediciones Khaaf.

Martínez García, I., \& Gil Flores, J. (2018). Explicación de la satisfacción en la dirección escolar a partir del desempeño de la función directiva. Education in the Knowledge Society, 19(1), pp. 77-95. doi: 10.14201/eks20181917795

Murillo, F. J., \& Krichesky, G. (2015). Mejora de la Escuela: medio siglo de lecciones aprendidas. REICE, Revista Iberoamericana sobre Calidad, Eficacia y Cambio en Educación, 13(1), pp. 69-102. Recuperado de https://revistas.uam.es/index.php/reice/article/view/2800/3015

Murillo, F. J., \& Martínez-Garrido, C. (2018). Factores de aula asociados al desarrollo integral de los estudiantes: un estudio observacional. Estudios Pedagógicos XLIV(1), pp. 181-205. doi: 10.4067/S0718-07052018000100181

Nair, P. (2013). Diseño de espacios educativos. Rediseñar las escuelas para centrar el aprendizaje en el alumno. Madrid: SM.

Roselló, M. (2010). El reto de planificar para la diversidad en una escuela inclusiva. Revista Iberoamericana de Educación, 51(4), pp. 1-10. Recuperado de https://rieoei.org/historico/deloslectores/3197Ramon.pdf

Sloep, P., \& Berlanga, A. (2011). Redes de aprendizaje, aprendizaje en red. Comunicar, XIX(37), pp. 55-64. doi: 10.3916/C37-2011-02-05

Stoll, L., \& Fink, D. (1999) Para cambiar nuestras escuelas. Reunir eficacia y mejora. Barcelona: Octaedro.

Sancho, J. M. (2018). Innovación y enseñanza. De la "moda" de innovar a la transformación de la práctica docente. Educaşão, 41(1), pp. 12-20. doi: 10.15448/1981-2582.2018.1.29523

Murillo, F. J., \& Muñoz-Repiso, M. (2020). La mejora de la escuela. Un cambio de mirada. Madrid: MECD/Octaedro.

Sancho, J. M. (2018). Innovación y enseñanza. De la "moda" de innovar a la transformación de la práctica docente. Educaçao, 41 (1), pp. 12-20. doi: 10.15448/1981-2582.2018.1.29523

Sun, J., \& Leithwood, K. (2014). Efectos del liderazgo escolar transformacional en el rendimiento de los estudiantes. Revista Iberoamericana sobre Calidad, Eficacia y Cambio en Educación, 12(4), pp. 41-70. Recuperado de https://revistas.uam.es/index.php/reice/article/view/2836/3053

Trujillo, F. (2018). Activos de aprendizaje. Utopias educativas en construcción. Madrid: SM.

Vergara, J. J. (2015). Aprendo porque quiero. Aprendizaje basado en proyectos, paso a paso. Madrid: SM. 